\title{
Dynamics of Electron-Induced Manipulation of Individual CO Molecules on $\mathrm{Cu}(111)$
}

\author{
L. Bartels, G. Meyer, and K.-H. Rieder \\ Institut für Experimentalphysik, Freie Universität Berlin, Arnimallee 14, 14195 Berlin, Germany \\ D. Velic, E. Knoesel, A. Hotzel, M. Wolf, and G. Ertl \\ Fritz-Haber-Institut der Max-Planck-Gesellschaft, Faradayweg 4-6, 14195 Berlin, Germany
}

(Received 10 September 1997)

\begin{abstract}
Electrons tunneling from a scanning tunneling microscope tip to individual $\mathrm{CO}$ molecules on $\mathrm{Cu}(111)$ can cause their hopping from the surface to the tip if the bias exceeds a threshold of $2.4 \mathrm{~V}$. Polarization- and time-resolved two-photon photoemission identifies the underlying elementary process as intermediate population of a $\mathrm{CO} 2 \pi^{*}$-derived level, which exhibits an ultrashort lifetime of $0.8-5 \mathrm{fs}$. From an isotope effect of $2.7_{-0.5}^{+0.3}$ it can be calculated that $\approx 0.05 \%$ of the tunneling current transiently occupies this level while a desorption of the excited molecule occurs only in $5 \times 10^{-9}$ of the cases. [S0031-9007(98)05403-9]

PACS numbers: 82.65.Pa, 61.16.Ch
\end{abstract}

The ultimate goal in surface dynamics is to obtain a fundamental understanding and to control elementary surface processes on a microscopic level. This requires both spatial and temporal resolution of such dynamical changes on an atomic scale. With the invention of the scanning tunneling microscope (STM) by Binnig and Rohrer [1] it has now become possible not only to observe but also to manipulate single atoms and molecules on surfaces $[2,3]$. Furthermore, tunneling spectroscopy (TS) allows one to obtain spectroscopic information with a spatial resolution on the atomic length scale [4]. Ultrafast laser techniques, on the other hand, provide direct insights into the dynamics of chemical reactions and energy transfer processes [5]. In particular, two-photon photoemission (2PPE) spectroscopy has become an important tool to probe the symmetry, energetics, and lifetimes of electronically excited states of atoms and molecules on metal surfaces on the femtosecond time scale [6,7]. In this Letter we demonstrate by combining these two powerful techniques, that STM and 2PPE are complementary in providing considerable insights into the excited state dynamics and the mechanism of stimulated desorption of single molecules on surfaces.

Recently, we presented a technique of transferring a CO molecule between the apex of a STM tip and a $\mathrm{Cu}(111)$ substrate (involving biases of up to $3 \mathrm{~V}$ ) and vice versa [8]. To our knowledge, this is the first system where reversible vertical transfer of a chemisorbed small molecule has been observed. Vertical manipulation of Xe atoms by vibrational heating [9] or electron scattering has been achieved by Eigler et al. [10]. For the STM-induced desorption of hydrogen from $\mathrm{Si}: \mathrm{H}$ two different excitation modes have been distinguished [11,12]: vibrational multiexcitation by high tunneling currents and electronic excitation by field emitted electrons from the STM tip. Desorption of $\mathrm{CO}$ from $\mathrm{Cu}(111)$ by ultrashort laser pulses of high energy has been reported by Prybyla et al. [13]. Their experiment involved heating of the substrate electronic temperature to several thousand $\mathrm{K}$ resulting in a de- sorption process induced by multiple electronic transitions [14] with a quantum yield of $\approx 10^{-3}$. Recently, Stipe et al. [15] presented impressive STM data on the dissociation of $\mathrm{O}_{2}$ by vibrational (multi)excitation of the intramolecular bond by tunneling electrons of low energy. In the following, we will show that the reversible transfer of $\mathrm{CO}$ molecules from the $\mathrm{Cu}(111)$ substrate to the STM tip is caused by attachment of a single electron into the $2 \pi^{*}$ orbital of CO. Time-resolved 2PPE clearly reveals the symmetry and line shape of this excited CO state and yields an upper and a lower limit for the lifetime. The excitation process shows a strong isotope effect. Evaluating these data within the 1D model proposed by Gadzuk [16] allows some conclusion on the lifetime of the excited state and its potential slope although the net quantum yield is of the order of $10^{-11}$. Using the standard Menzel-Gomer-Redhead (MGR) model $[17,18]$ these data can be used to gain an estimation of the partial current flowing through the $2 \pi^{*}$ state, which is of great interest for comparison with STM currents calculated within the electron quantum scattering technique [19].

For all STM measurements we use a self-built low temperature STM [20] operating at $15 \mathrm{~K}$ [21]. Samples are prepared by several cycles of sputtering with $\mathrm{Ne}^{+}$, annealing to $\simeq 400{ }^{\circ} \mathrm{C}$ and subsequent deposition of below $1 \%$ of a $\mathrm{CO}$ monolayer (ML) [22] at $15 \mathrm{~K}$. Excitation of single $\mathrm{CO}$ molecules is performed by positioning the STM tip exactly above the molecule at a sample bias $V$ of $2 \mathrm{~V}$. Subsequently, the bias is switched to a minimum of $2.4 \mathrm{~V}$; this results in a tunneling current $I$ of a few nA. Within a few seconds, a clear change in the simultaneously recorded $d I / d V$ can be observed. It is caused by a hop of the adsorbed molecule either to the tip apex or to an adsite on the sample a few angstroms away (top part of Fig. 1) with $\mathrm{a} \simeq 1: 3$ probability.

The mean time before a hop of the adsorbed CO molecule is induced ("hopping time") depends on the current and bias used. The threshold bias to induce hops resulting 


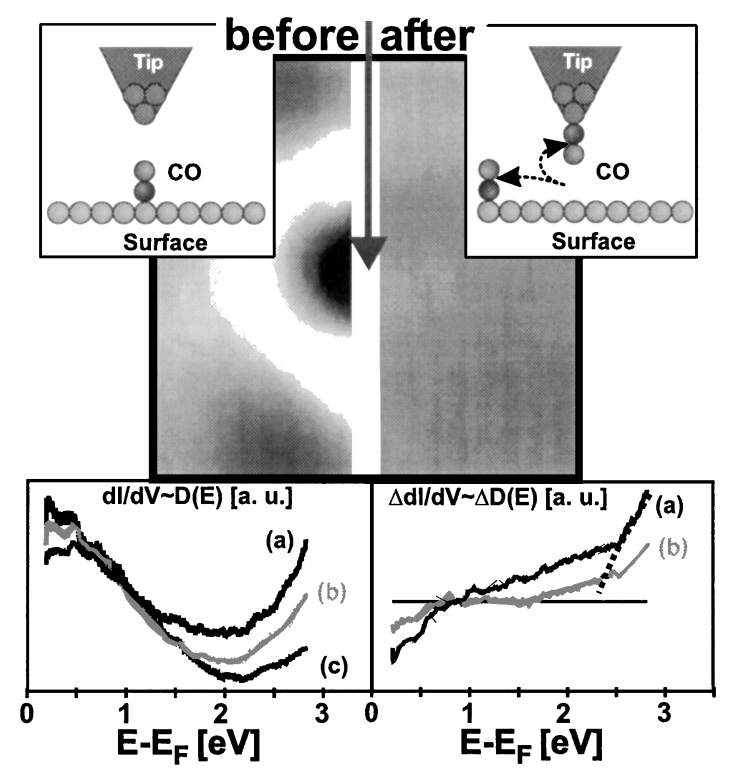

FIG. 1. Center: STM image of a single CO molecule on $\mathrm{Cu}(111)$ taken with a bare (metallic) tip (top left sketch) at $2 \mathrm{~V}$ and $1.5 \mathrm{nA}$ column by column starting from the left. As the center column of the image is reached, the tip is stopped at its center and the bias switched to $2.7 \mathrm{~V}$ for $3.8 \mathrm{~s}$. During this time an abrupt change of the simultaneously recorded $I$ and $d I / d V$ occurred, which can be interpreted as hopping of the CO molecule away from its initial site either to the tip apex or to another adsite nearby (top right sketch). Hence, as the scanning is resumed, it could not be found at its initial position on the sample (image size: $25 \AA \times 25 \AA$ ). Performing tunneling spectroscopy at the center of the CO molecule (a), at the edge of the image (c), and in between (b) - instead of inducing a hop - the three $d I / d V$ spectra (bottom left diagram) can be found. The difference spectra approximately reveal the CO-induced LDOS (bottom right diagram), which shows a rise above $\approx 1 \mathrm{~V}$ followed by a steep increase starting at $\simeq 2.4 \mathrm{~V}$ (dashed line).

in both lateral and vertical transfer is $2.4 \mathrm{~V}$. Thus, in the following both kinds of hops are treated as originating from the same excitation process. In the whole bias range used for vertical manipulation (up to $3 \mathrm{~V}$ ) [8] the dependence of the hopping rate on the current at a fixed bias is clearly linear (see Fig. 2). The net quantum yield per tunneling electron $P_{\text {tot }}$ rises from zero below $2.4 \mathrm{~V}$ to $2.7 \times 10^{-11}$ at $2.7 \mathrm{~V}$ and $3.0 \times 10^{-11}$ at $3.0 \mathrm{~V}$. The linear dependence of the rate on the tunneling current clearly indicates that a single electron process is responsible for the observed jumps. A comparison of $d I / d V$ curves of TS acquired at different lateral distances from an adsorbed $\mathrm{CO}$ molecule clearly shows a CO-induced increase of $d I / d V$ at biases above $\simeq 1 \mathrm{~V}$ relative to the TS on the clean surface (Fig. 1, bottom part). Above $2.4 \mathrm{~V}$ another, much steeper increase is observed (indicated by the dashed line), which coincides with the bias threshold to induce hops of $\mathrm{CO}$ molecules. The $d I / d V$ signal is approximately proportional to the local density of states (LDOS) of the sample [23]. Thus, the hopping of the $\mathrm{CO}$ molecules has to be attributed to population of a CO-derived part of the LDOS with electrons from the STM tip as indicated in the bottom part of Fig. 4.

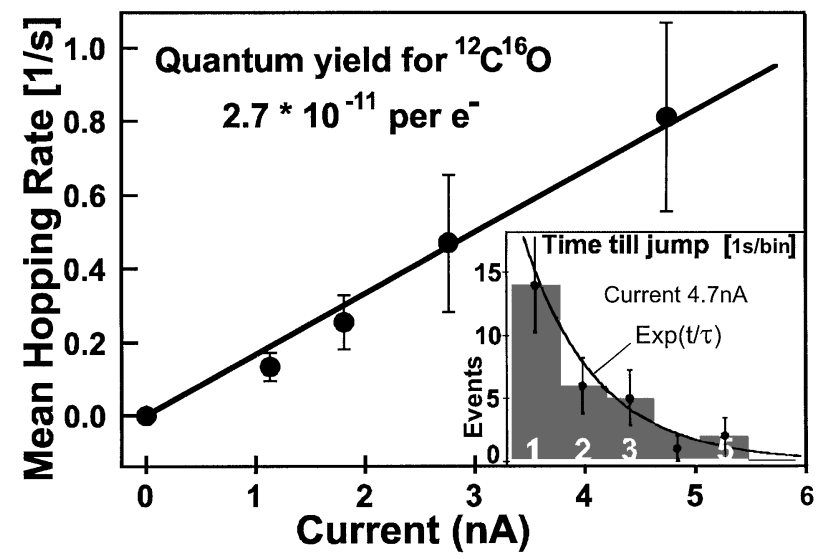

FIG. 2. Mean hopping rate of "normal" ${ }^{12} \mathrm{C}^{16} \mathrm{O}$ molecules at $2.7 \mathrm{~V}$ bias versus current. The rates were calculated from the distribution of hopping times, which is plotted for $2.7 \mathrm{~V}$ bias and $4.7 \mathrm{nA}$ current in the inset. The error bars indicate the error of the exponential fit of the hopping times.

As neither the symmetry of the CO-induced state can be resolved by TS nor the high energy side of the state can be probed without inducing a hop of the adsorbed molecule [and possible interference with the $\mathrm{Cu}(111)$ image state] we have performed $2 \mathrm{PPE}$ [6] at CO coverages of $\frac{1}{3} \mathrm{ML}$ resulting in the $\sqrt{3} \times \sqrt{3}$ phase of $\mathrm{CO} / \mathrm{Cu}(111)$. This phase consists entirely of $\mathrm{CO}$ molecules adsorbed on the on-top sites [24]. Spectra at a photon energy of $4.1 \mathrm{eV}$ clearly show that the steep increase in the TS corresponds to the onset of the $2 \pi^{*}$-derived state of $\mathrm{CO} / \mathrm{Cu}(111)$ (left part of Fig. 3). Its center is found to be at $3.5 \mathrm{eV}$ with a FWHM of $\simeq 870 \mathrm{meV}$. From time-resolved $2 \mathrm{PPE}$ measurements with $45 \mathrm{fs}$ pulses an upper limit of the relaxation time of the excited state of $5 \mathrm{fs}$ is obtained

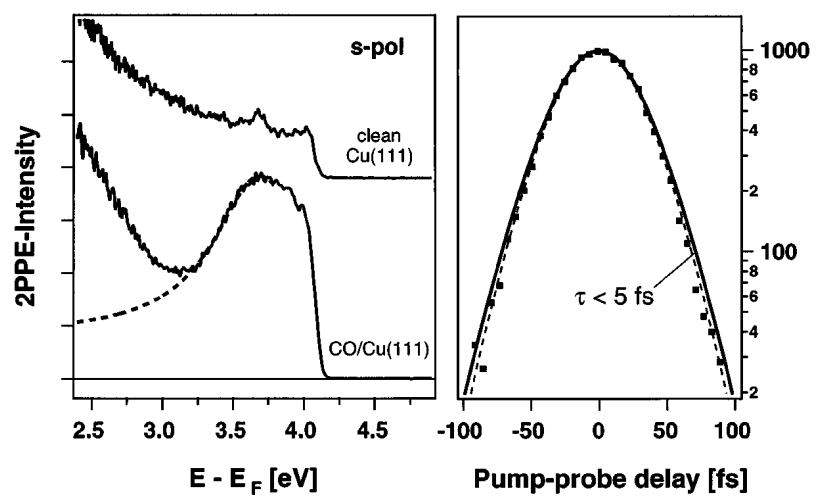

FIG. 3. Left part: 2PPE spectra obtained with $s$-polarized $4.1 \mathrm{eV}$ photons on clean $\mathrm{Cu}(111)$ and on $(\sqrt{3} \times \sqrt{3})$ $\mathrm{CO} / \mathrm{Cu}(111)$. They show a $\mathrm{CO}$-induced state at $3.5 \mathrm{eV}$ with a FWHM of the onset of $0.8 \mathrm{eV}$. Because of its appearance at $s$ polarization it can be identified as the $2 \pi^{*}$ state of $\mathrm{CO} / \mathrm{Cu}(111)$. The dashed curves indicate a Lorenzian fit of the onset of the $2 \pi^{*}$ state which coincides with the enhancement of LDOS observed in the TS. Right part: Autocorrelation trace derived from a pump probe experiment of the signal from the $2 \pi^{*}$ state. From the measured data (dots) a maximum lifetime of $5 \mathrm{fs}$ (solid line) can be deduced. The dashed line indicates the experimental resolution. 
(right part of Fig. 3), whereas the spectral width of the $2 \pi^{*}$ state indicates a lower limit of $0.8 \mathrm{fs}$. This extremely short excited state lifetime agrees well with the extremely small quantum yield of the STM-induced transfer process.

The CO-Cu stretch has a vibrational energy of $42 \mathrm{meV}$ [25] and the binding energy of $\mathrm{CO}$ to $\mathrm{Cu}(111)$ is $425 \mathrm{meV}$ [26]. The tunneling gap distance is $\approx 7 \AA$ [27]. Approximating the potential curve of the adsorbed $\mathrm{CO}$ molecule with a Morse potential we find that there is virtually no overlap between the adsorption potential on the substrate and on the tip (top part of Fig. 4), even if a slightly higher binding energy of the molecule on the tip is assumed. Hence, the STM-induced transfer of the CO molecule between the substrate and the STM tip can be discussed in terms of a desorption process. The electric field of $\approx 3 \mathrm{~V} / \mathrm{nm}$ at the position of the adsorbed molecule is small compared to the minimum field of more than $10 \mathrm{~V} / \mathrm{nm}$ needed to desorb $\mathrm{CO}$ molecules in field ion microscopy experiments [28]. In a calculation of the effect of vibrational (multi)excitation of the $\mathrm{CO}-\mathrm{Cu}$ bond by dipole interaction with the field of tunneling electrons using the model by Avouris et al. [9] an effective temperature of the bond of $<40 \mathrm{~K}$ can be estimated, which is far too low to play a major role in the desorption process.

Thus, we conclude that the hopping process is induced by a single electronic transition of a $\mathrm{CO}$ molecule from the ground state to the excited state potential $V_{2 \pi^{*}}$ via attachment of a tunneling electron into the $\mathrm{CO} 2 \pi^{*}$ state (Fig. 4). Desorption processes induced by electronic

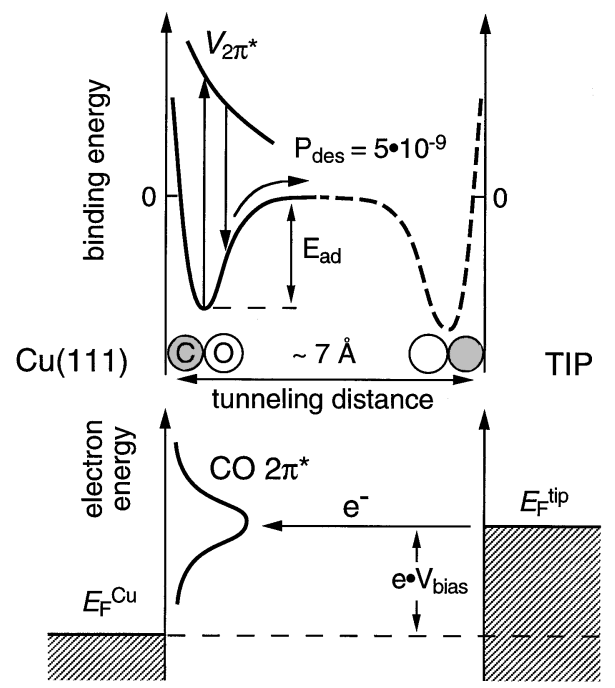

FIG. 4. Top part: potential curve of the bound state of a CO molecule between the $\mathrm{Cu}(111)$ substrate (left) and the STM tip (right). A higher binding energy on the tip is assumed. Virtually no overlap between the two potential wells occurs. Additionally the assumed potential curve $V_{2 \pi^{*}}$ of the excited state is shown. The desorption process according to the MGR model is indicated with arrows. It has a yield per excitation of $P_{\text {des }}=5 \times 10^{-9}$. Bottom part: At positive sample bias $V_{\text {bias }}$ tunneling electrons from the STM tip (right) can transiently occupy states of the sample between $E_{F}$ and $e^{-} V_{\text {bias. }}$. Thus, at $2.4 \mathrm{~V}$ bias the electrons start tunneling into the $2 \pi^{*}$ state. excitation have been discussed within the framework of the MGR model [17,18]. In this model a pronounced isotope effect is expected for a system with an ultrashort excited state lifetime [29]. From such an isotope effect the excitation probability per incoming electron (i.e., the fraction of electrons which transiently occupy the $2 \pi^{*}$ state) can be calculated without any assumption about the value of the $2 \pi^{*}$ lifetime.

From calculations of Bocquet and Sautet [30] it is known that the tunneling current passes only partially through electronic states attributed to the adsorbed molecule even if the tip is positioned in its direct vicinity. Furthermore, a whole number of electronic states of the substrateadsorbate system are involved in the transition of electrons from the tip shaft to the bulk of the sample. Therefore, the fractional tunneling current through the $\mathrm{CO} 2 \pi^{*}$ state is of great interest. In order to determine this fraction, STM experiments on surfaces with similar amounts of ${ }^{12} \mathrm{C}^{16} \mathrm{O}$ and ${ }^{13} \mathrm{C}^{18} \mathrm{O}$ in the mML regime were performed in the following fashion: A pair of $\mathrm{CO}$ molecules was arbitrarily chosen, resulting in a $50 \%$ chance of picking two different isotopomers. The two molecules of the pair were alternatingly excited to perform hops. To ensure inducing hops on exactly the chosen molecules, a minimum of $2 \mathrm{~nm}$ distance between the probed molecule and its nearest neighbor is kept by, if necessary, laterally translating the molecule with the STM tip before exciting it. These measurements inherently combine the unique ability of the STM to image on the atomic scale (to find the position of a molecule initially and after the excitation) and its application as a manipulation tool on the same minute scale (to induce a hop on a specified molecule and to clear a molecule from too close neighbors). By this method of alternating excitations an impact of tip changes on the measurement can be ruled out. From the thus acquired distributions of hopping times for different pairs of molecules a hopping time for the lighter species could be obtained, which agrees within $20 \%$ with the hopping time observed for ${ }^{12} \mathrm{C}^{16} \mathrm{O}$ molecules. However, the average hopping time of the heavier species comes out to be 2.7 times longer. Thus, an isotope effect of $\approx 2.7_{-0.5}^{+0.3}$ can be estimated. A detailed description of the data evaluation will be published elsewhere. Using this isotope effect acquired on lateral and vertical hops in the 1D MGR model we find that $0.5 \%(0.01 \%-4 \%)$ of the tunneling current is passing through the $2 \pi^{*}$ level and the hopping rate per excitation is $\approx 5 \times 10^{-9}$. Such a minute desorption probability cannot be resolved with most photostimulated desorption techniques without considerable substrate heating.

Gadzuk [16] proposed a semiclassical approach to calculate the desorption probability of a Gaussian wave packet on a one-dimensional potential energy surface. The lifetime of the electron in the $2 \pi^{*}$ state and the slope of the excited state potential curve enter as parameters. Using this model the desorption probability per electronic excitation $P_{\text {des }}$ in dependence on the mass of the desorbing species can be obtained. Applying this method to ${ }^{12} \mathrm{C}^{16} \mathrm{O}$ 


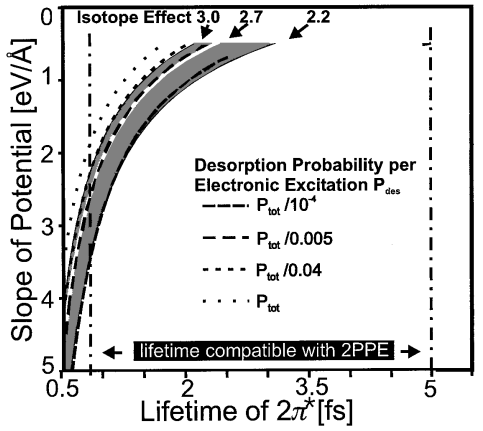

FIG. 5. The gray areas show the possible range for the lifetime and the potential gradient of the $2 \pi^{*}$ state of $\mathrm{CO} / \mathrm{Cu}(111)$, calculated from the isotope effect within the model proposed by Gadzuk [16]. The white line between the gray areas corresponds to the isotope effect of 2.7. Lines of equal desorption probability $P_{\text {des }}$ per electronic excitation are indicated for the desorption probabilities $P_{\text {des }}$ calculated from the MGR model for the isotope effect of $2.7_{-0.5}^{+0.3}$ and for the desorption probability $P_{\text {tot }}$ resulting from the total STM current flowing through the $2 \pi^{*}$ state of $\mathrm{CO}$.

and ${ }^{13} \mathrm{C}^{18} \mathrm{O}$ on $\mathrm{Cu}(111)$ and using the observed isotope effect of $2.7_{-0.5}^{+0.3}$ (gray area in Fig. 5) and the measured limits of the lifetime (0.8-5 fs) of the excited state (dash-dotted vertical lines in Fig. 5), we find that the potential slope of the excited state at the ground state $\mathrm{CO}-\mathrm{Cu}$ equilibrium distance is confined to a small range $(\simeq 1 \mathrm{eV}$ per $\AA$ ). Additionally, we plotted the calculated contours of fixed desorption probability per excitation at the values obtained from the MGR model in the same diagram (dashed lines in Fig. 5) and find impressive agreement between both models. To our knowledge, this is the first time that information about the shape and lifetime of an excited state potential shape of single adsorbates could be gained from STM and 2PPE measurements. Nevertheless, it seems to be a worthwhile aim to find a multidimensional picture of the hopping process, which can explain the distribution of hopping directions and eventually shed more light on the underlying microscopic process.

In summary, we found that vertical manipulation of $\mathrm{CO}$ molecules on $\mathrm{Cu}(111)$ by a STM tip originates from a single electron excitation process of the $\mathrm{CO}-2 \pi^{*}$ resonance, which is identified by polarization and timeresolved 2PPE and exhibits a lifetime of $\leq 5 \mathrm{fs}$. The hopping process has an ultralow yield and shows a strong isotope effect of $2.7_{-0.5}^{+0.3}$. Using these data a number of dynamical parameters of the $\mathrm{CO}-\mathrm{Cu}(111)$ system can be deduced including the fraction of the tunneling current passing through the $2 \pi^{*}$ state, the shape of excited state potential surface, and the lifetime of the excited state. Remarkably, the consistency of our results obtained within the framework of the MGR model suggests that the Born Oppenheimer approximation, at its heart, is an applicable concept even in the regime of a few fs, and that tunneling electrons can be treated in this framework.

We gratefully acknowledge financial support by the DFG (No. RI 472/3-1) and FES (L. B.).
[1] G. Binnig, H. Rohrer, Ch. Gerber, and E. Weibel, Phys. Rev. Lett. 49, 52 (1982).

[2] D. M. Eigler and E. K. Schweitzer, Nature (London) 344, 524 (1990).

[3] G. Meyer, L. Bartels, S. Zöphel, E. Henze, and K.-H. Rieder, Phys. Rev. Lett. 78, 1512 (1997).

[4] R. S. Becker, J. A. Golovchenko, D. R. Hamann, and B. S. Swartzentruber, Phys. Rev. Lett. 55, 2028 (1985).

[5] A. H. Zewail, Femtochemistry-Ultrafast Dynamics of the Chemical Bond (World Scientific, Singapore, 1994).

[6] M. Wolf, Surf. Sci. 377, 343 (1996).

[7] M. Bauer, S. Pawlik, and M. Aeschlimann, Phys. Rev. B 55, 10040 (1997).

[8] L. Bartels, G. Meyer, and K.-H. Rieder, Appl. Phys. Lett. 71, 213 (1997).

[9] R.E. Walkup, D. M. Newns, and Ph. Avouris, J. Electron Spectrosc. Relat. Phenom. 64/65, 523 (1993).

[10] D. M. Eigler, C. P. Lutz, and W.E. Rudge, Nature (London) 352, 600 (1991).

[11] R. S. Becker, G. S. Higashi, Y. J. Chabal, and A. J. Becker, Phys. Rev. Lett. 65, 1917 (1990).

[12] T.-C. Shen, C. Wang, G.C. Abeln, J.R. Tucker, J.W. Lyding, Ph. Avouris, and R. E. Walkup, Science 268, 1590 (1995).

[13] J.A. Prybyla, H.W.K. Tom, and G.D. Aumiller, Phys. Rev. Lett. 68, 503 (1992).

[14] J. Misewich, T.F. Heinz, and D. M. Newns, Phys. Rev. Lett. 68, 3737 (1992).

[15] B. C. Stipe, M. A. Rezaei, W. Ho, S. Gao, M. Persson, and B. I. Lundquist, Phys. Rev. Lett. 78, 4410 (1997); B.C. Stipe, M. A. Rezaei, and W. Ho, J. Chem. Phys. 107, 6443 (1997).

[16] J. W. Gadzuk, Surf. Sci. 342, 345 (1995).

[17] D. Menzel and R. Gomer, J. Chem. Phys. 41, 3311 (1964).

[18] P. A. Redhead, Can. J. Phys. 42, 886 (1964).

[19] P. Sautet and C. Joachim, Phys. Rev. B 38, 12238 (1988).

[20] G. Meyer, Rev. Sci. Instrum. 67, 2960 (1996).

[21] Although electrochemically etched tungsten tips were used, we expect them to be $\mathrm{Cu}$ covered due to occasional tip-substrate contact during final in situ tip preparation. Extensive care was taken to prevent tip artifacts in all measurements. Tips used exhibited metallic imaging quality and consistent spectra of the occupied substrate states.

[22] One CO molecule per substrate unit cell.

[23] R. M. Feenstra, J.A. Stroscio, and A. P. Fein, Surf. Sci. 181, 295 (1987).

[24] In contrast to previous work at higher coverages, where CO is adsorbed on different adsites: E. Knoesel, T. Hertel, M. Wolf, and G. Ertl, Chem. Phys. Lett. 249, 409 (1995).

[25] R. Raval, S.F. Parker, M.E. Pemble, P. Hollins, J. Pritchard, and M. A. Chester, Surf. Sci. 203, 353 (1988).

[26] W. Kirstein, B. Krüger, and F. Thieme, Surf. Sci. 176, 505 (1986).

[27] Measured on the bare surface by successively pushing the STM closer to the substrate until a mechanical change of the tip and the substrate occurred.

[28] Yu. Suchorski (private communication).

[29] Which is well known from electron stimulated desorption studies, see, e.g., R. D. Ramsier and J.T. Yates, Surf. Sci. Rep. 12, 243 (1991).

[30] M.-L. Bocquet and P. Sautet, Surf. Sci. 360, 128 (1996). 